\title{
The longitudinal-transverse bending of reinforced concrete rods
}

\author{
Yury Nemirovskii ${ }^{1}$, and Sergey Tikhonov ${ }^{2, *}$ \\ ${ }^{1}$ Khristianovich Institute of Theoretical and Applied Mechanics Siberian Branch of the Russian \\ Academy of Sciences, Physics of Fast Processes Laboratory, 630090 Institutskaya str., 4/1, \\ Novosibirsk, Russia \\ ${ }^{2}$ I. Ulianov Chuvash State University, Faculty of Information and Computer Systems, 428015 \\ Moskovskiy pr., 14, Cheboksary, Russia
}

\begin{abstract}
The longitudinal transverse bending of multilayer rods made of reinforced concrete of arbitrary cross-section is considered. It is assumed that the coefficients in the connection equation between stresses, strains and temperature are different in each layer. The achievement of maximum deformation of the permissible limit value at stretching or compression is accepted as the criterion of conditional limit state in the i-layer. The case of longitudinal-transverse bending of a hinged rod is considered as an example of this method of solution. The distribution of bending moments and longitudinal forces, displacements and deformations is determined.
\end{abstract}

\section{Introduction}

The active development of mass housing construction requires the expansion of the industrial nomenclature of building materials and structures, the use of new technologies in construction and the creation of new durable and economically effective building structures. Currently, reinforced concrete rod elements are the most effective elements of bearing structures for the frameworks of housing and industrial elements. Reinforced concrete, due to its physical and mechanical properties, durability and technical and economic efficiency of production and use of products from them, as well as the availability of sufficient raw materials for cement and concrete over the last decades and in the long term has been and will remain the basis of any construction project.

Significant improvement in the quality of concrete based products can be achieved by reinforcing with high-modulus and high-strength fibers of metal, polymer or stone (when creating the poly-reinforced structures), as well as using rubber and polymer concrete [1-5]. An important problem in the design of such elements is economy of expense and cost of the materials. This circumstance causes the necessity of a unified approach to the calculation and analysis of the behavior of hybrid structures with different symmetric and asymmetric cross-section profiles and different reinforcement structures.

\footnotetext{
* Corresponding author: strangcheb@mail.ru
} 


\section{Methodology}

The considered structures belong to the class of layered-fibrous rods with significantly different properties in the layers, which should be determined by special experiments and taken into account in the developed calculation method.

Eventually, such method should be sufficiently reliable, relatively simple and flexible to calculate a wide range of varying possibilities for the conditions of fastening, loading, structural forms of sections and reinforcement parameters. For structures operating under transverse bending conditions, the corresponding solutions were obtained in the works [1, $3,4,6,7]$. For conditions of longitudinal-transverse bending, there are no corresponding solutions.

We will take into account an important feature of concrete and reinforced concrete elements - significantly different regularities of deformation under conditions of stretching and compression in developing the design scheme. Concretes (reinforced and unreinforced) at stretching behave as linear-elastic, while at compression the diagrams show significant nonlinearity even at low loading levels. This fact should be taken into account, as in the considered structures the areas with various deformation laws are not known in advance and change during the loading of structures. Therefore, uniform rather simple approximations of deformation diagrams with different (by zones) coefficients are advisable to use in different cross-section zones for the construction of uniform resolving equations, as well as in works $[1,3]$. Then the relationship between stresses $\sigma_{i}$, strains $\varepsilon$ and temperature $T$ in the $i$-zone of the rod section $S=\sum_{i=1}^{n} S_{i}$ (Figure 1) is represented in the form

$$
\sigma_{i}=A_{1 i}\left(\varepsilon-d_{i} T\right)+A_{2 i}\left(\varepsilon-d_{i} T\right)^{2}
$$

where $A_{1 i}, A_{2 i}$ are experimentally found coefficients of materials of the $i$-layer (depending on the layer structure and properties of phase materials), $d_{i}$ are coefficients of thermal expansion. Later, for the simplicity of statement, we content ourselves with the case $n=3$.

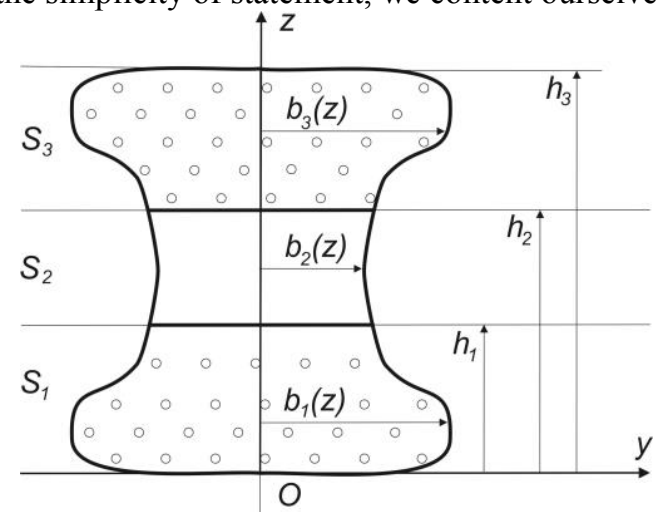

Fig. 1. Cross-section of a multilayer beam

The loss of operational qualities of reinforced concrete structures, as noted in many experimental studies $[1,4-11]$ is characterized by limit deformations $\varepsilon_{*_{i}}^{-}$(under compression) and $\varepsilon_{*_{i}}^{+}$(under tension). Their excess usually leads to intense fracture processes [11, 12]. Then, the achievement of maximum deformation of the limit permissible value under tension or compression is taken as a criterion of the conditional 
limit state in the $i$-layer, that is the dependences (1) are determined on the section $-\varepsilon_{*_{i}}^{-} \leq \varepsilon \leq \varepsilon_{*_{i}}^{+}$.

Thus, for the material samples of this layer, the conditions are satisfied with sufficient accuracy

$$
\sigma_{i}\left(\varepsilon_{*_{i}}^{+}\right)=\sigma_{*_{i}}^{+}, \quad \sigma_{i}\left(\varepsilon_{*_{i}}^{-}\right)=-\sigma_{*_{i}}^{-},\left.\quad \frac{d \sigma_{i}}{d \varepsilon}\right|_{\varepsilon_{i}=-\varepsilon_{i^{*}}^{-}}=0,
$$

where $\sigma_{*_{i}}^{+}, \sigma_{*_{i}}^{-}$are strength limits under tension and compression of samples of the $i$-layer.

Then, for the coefficients $A_{1 i}, A_{2 i}$ we have the expressions

$$
A_{1 i}=2 \frac{\sigma_{*_{i}}^{-}}{\varepsilon_{*_{i}}^{+}}\left(\sqrt{1+\frac{\sigma_{*_{i}}^{+}}{\sigma_{*_{i}}^{-}}}-1\right), \quad A_{2 i}=\frac{A_{1 i}^{2}}{4 \sigma_{*_{i}}^{-}} .
$$

We choose the Cartesian coordinate system with the axis $x$ directed along the axis of the rod with a definite reference to the cross section, the vertically upward axis $z$ (the deflection axis) and with the beginning of coordinates at one of the ends of the rod.

To simplify the further obtained formulas, we assume that the structures of hybrid reinforced rods and the distribution of external loads and temperatures are coordinated, so that in deformation process the effects of twisting either do not occur at all, or they are so insignificant that they can be neglected. Then the arising stress fields $\sigma_{j}$ will produce internal force factors

$$
N=\sum_{i=1}^{n} \int_{S_{i}} \sigma_{i} d S, M_{y}=-\sum_{i=1}^{n} \int_{S_{i}} \sigma_{i} z d S,
$$

which must satisfy the equilibrium equations

$$
\frac{d^{2} M_{y}}{d x^{2}}=q_{z}-\frac{d m_{y}}{d x}, \frac{d N}{d x}=-q_{x} .
$$

Here $N$ is projection of the internal force vector on the axis $x, M_{y}$ are projections of the internal moment vector on the axis $y$. The values $q_{x}, q_{z}$ are projections of the vector of distributed load applied to the axis of the rod, $m_{y}$ is projection of the vector of distributed moment on the axis $y$.

Integrating equations (4), we obtain

$$
N(x)=N(0)-\int_{0}^{x} q_{x} d x, \quad M_{y}(x)=M_{y}(0)-Q_{z}(0) x-\int_{0}^{x} m_{y} d x+\int_{0}^{x}\left[\int_{0}^{x} q_{z} d x\right] d x .
$$

In the case of statically determined rods, the values $N(0), Q_{z}(0), M_{y}(0)$ are determined from the equilibrium conditions of the extreme knots. For statically indefinable problems it will be necessary to make additional conditions of compatibility of deformations.

Using the Kirchhoff classical kinematic hypotheses, for deformations we will have the expressions

$$
\begin{aligned}
& \varepsilon(x, y, z)=\varepsilon_{0}(x)-y \kappa_{z}(x), \\
& \varepsilon_{0}(x)=\frac{d u_{0}}{d x}, \kappa_{z}=-\frac{d^{2} w_{0}}{d x^{2}},
\end{aligned}
$$

where $u_{0}, w_{0}$ are components of the displacement vector of points of axial line of the rod.

Integrating the relations (7), we obtain

$$
u_{0}(x)=u_{0}(0)+\int_{0}^{x} \varepsilon_{0}(x) d x, \quad w_{0}(x)=w_{0}(0)+\varphi_{z}(0) x+\int_{0}^{x}\left[\int_{0}^{x} \kappa_{z}(x) d x\right] d x,
$$




$$
\varphi_{z}(x)=\varphi_{z}(0)+\int_{0}^{x} \kappa_{z}(x) d x .
$$

The values $u_{0}(0), w_{0}(0), \varphi_{z}(0)$ are found from the condition of fastening the rod.

Substituting the expressions (1) in (3) and taking into account the actual structure of arrangement of the materials in the cross section in the case of the dependence of the stationary temperature field on the longitudinal coordinate $x$ for the quantities $N, M_{y}$

$$
N=2 \sum_{i=1}^{3} \iint_{S} \sigma_{i} d S=f_{0}+f_{1} \varepsilon_{0}(x)+f_{2} \kappa_{z}(x)+f_{11} \varepsilon_{0}^{2}(x)+f_{22} \kappa_{z}^{2}(x)+f_{12} \varepsilon_{0}(x) \kappa_{z}(x),
$$

where

$$
\begin{gathered}
f_{0}=2 \sum_{i=1}^{3}\left(A_{2 i} d_{i} T^{2}-A_{1 i} d_{i} T\right) \int_{h_{i-1}}^{h_{i}} b_{i}(z) d z, f_{1}=2 \sum_{i=1}^{3}\left(A_{1 i}-2 A_{2 i} d_{i} T\right) \int_{h_{i-1}}^{h_{i}} b_{i}(z) d z, \\
f_{2}=\sum_{i=1}^{3}\left(2 A_{2 i} d_{i} T-A_{1 i}\right) \int_{h_{i-1}}^{h_{i}} b_{i}^{2}(z) d z, f_{11}=2 \sum_{i=1}^{3} A_{2 i} \int_{h_{i-1}}^{h_{i}} b_{i}(z) d z, f_{22}=\frac{2}{3} \sum_{i=1}^{3} A_{2 i} \int_{h_{i-1}}^{h_{i}} b_{i}^{3}(z) d z, \\
f_{12}=-2 \sum_{i=1}^{3} A_{2 i} \int_{h_{i-1}}^{h_{i}} b_{i}^{2}(z) d z \\
\left.g_{0}=-2 \sum_{i=1}^{3} \iint_{S}^{3} z \sigma_{i} d S=g_{0}+g_{1} \varepsilon_{0}(x)+g_{2} \kappa_{z}(x)+g_{11} T^{2}-A_{1 i}(x)+g_{i} T\right) \int_{h_{i-1}}^{2} z b_{i}(z) d z, \kappa_{z}^{2}(x)+g_{12} \varepsilon_{0}(x) \kappa_{z}(x),\left(1 \sum_{i=1}^{3}\left(A_{1 i}-2 A_{2 i} d_{i} T\right) \int_{h_{i-1}}^{h_{i}} z b_{i}(z) d z,\right. \\
g_{2}=-2 \sum_{i=1}^{3}\left(2 A_{2 i} d_{i} T-A_{1 i}\right) \int_{h_{i-1}}^{h_{i}} z b_{i}^{2}(z) d z, g_{11}=-2 \sum_{i=1}^{3} A_{2 i} \int_{h_{i-1}}^{h_{i}} z b_{i}(z) d z, \\
g_{22}=-\frac{2}{3} \sum_{i=1}^{3} A_{2 i} \int_{h_{i-1}}^{h_{i}} z b_{i}^{2}(z) d z, g_{12}=2 \sum_{i=1}^{3} A_{2 i} \int_{h_{i-1}}^{h_{i}} z b_{i}^{2}(z) d z, h_{0}=0 .
\end{gathered}
$$

\section{Results}

We consider the case of longitudinal-transverse bending of a rod of length 1 (Figure 2) as an example of this method of solution.

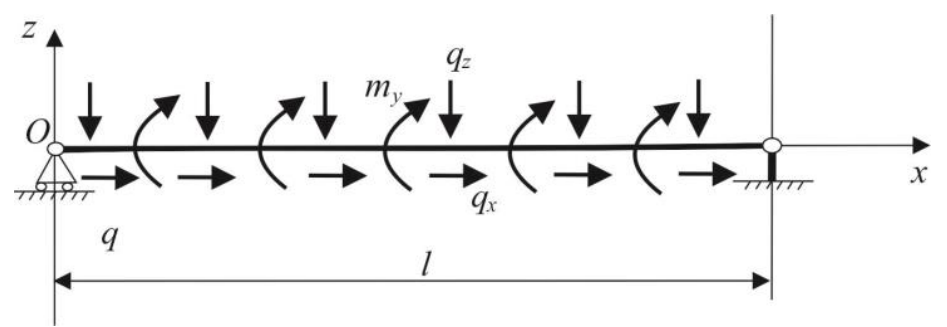

Fig. 2. Longitudinal-transverse bending of the rod of length $l$. 
We assume that the rod is under the distributed loads and bending moments

$$
q_{z}(x)=q_{0 z}\left(\alpha_{1}+\alpha_{2} x\right), q_{x}(x)=q_{0 x} \frac{x}{l}, m_{y}(x)=m_{0} \frac{x}{l},
$$

where $q_{0 x}, q_{0 z}, m_{0}, \alpha_{1}, \alpha_{2}-$ const.

From conditions of fastening of the rod, we have

$$
N(0)=M(0)=M(l)=0,
$$

Then, from (4), (12), (13) we obtain the distribution of bending moment and longitudinal force

$$
N=-q_{0 x} \frac{x}{l}, \quad M_{y}=\left(\frac{m_{0}}{2}-q_{0 z}\left(\alpha_{1} \frac{l}{2}+\alpha_{2} \frac{l^{2}}{6}\right)\right) x-\frac{m_{0}}{2 l} x^{2}+q_{0 z}\left(\frac{\alpha_{1}}{2} x^{2}+\frac{\alpha_{2}}{6} x^{3}\right) .
$$

We assume that the cross-section of the rod has the form shown in Figure 3.

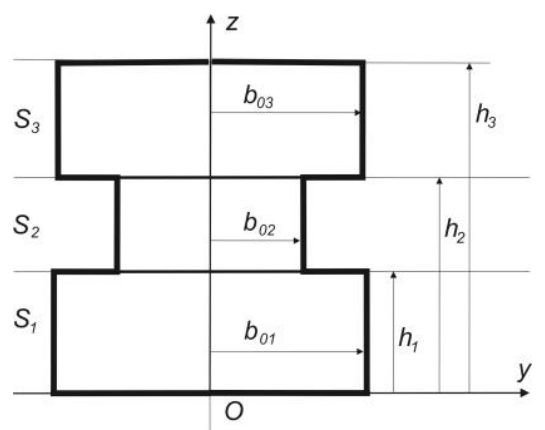

Fig. 3. Cross-section of the rod

Then from (7), (10), (11), (14) we obtain a system of differential equations for determining the deformed state

$$
\begin{gathered}
f_{1} \frac{d u_{0}}{d x}-f_{2} \frac{d^{2} w_{0}}{d x^{2}}+f_{11}\left(\frac{d u_{0}}{d x}\right)^{2}+f_{22}\left(\frac{d^{2} w_{0}}{d x^{2}}\right)^{2}-f_{12} \frac{d u_{0}}{d x} \frac{d^{2} w_{0}}{d x^{2}}+f_{0}+q_{0 x} \frac{x}{l}=0 \\
g_{1} \frac{d u_{0}}{d x}-g_{2} \frac{d^{2} w_{0}}{d x^{2}}+g_{11}\left(\frac{d u_{0}}{d x}\right)^{2}+g_{22}\left(\frac{d^{2} w_{0}}{d x^{2}}\right)^{2}-g_{12} \frac{d u_{0}}{d x} \frac{d^{2} w_{0}}{d x^{2}}+g_{0}+ \\
+\left(\frac{m_{0}}{2}-q_{0 z}\left(\alpha_{1} \frac{l}{2}+\alpha_{2} \frac{l^{2}}{6}\right)\right) x-\frac{m_{0}}{2 l} x^{2}+q_{0 z}\left(\frac{\alpha_{1}}{2} x^{2}+\frac{\alpha_{2}}{6} x^{3}\right)=0
\end{gathered}
$$

where

$$
f_{0}=2 \sum_{i=1}^{3} b_{0 i}\left(h_{i}-h_{i-1}\right)\left(A_{2 i} d_{i} T^{2}-A_{1 i} d_{i} T\right), \quad f_{1}=2 \sum_{i=1}^{3} b_{0 i}\left(h_{i}-h_{i-1}\right)\left(A_{1 i}-2 A_{2 i} d_{i} T\right),
$$




$$
\begin{gathered}
f_{2}=\sum_{i=1}^{3} b_{0 i}^{2}\left(h_{i}-h_{i-1}\right)\left(2 A_{2 i} d_{i} T-A_{1 i}\right), f_{11}=2 \sum_{i=1}^{3} b_{0 i}\left(h_{i}-h_{i-1}\right) A_{2 i}, \\
f_{22}=\frac{2}{3} \sum_{i=1}^{3} b_{0 i}^{3}\left(h_{i}-h_{i-1}\right) A_{2 i}, f_{12}=-2 \sum_{i=1}^{3} b_{0 i}^{2}\left(h_{i}-h_{i-1}\right) A_{2 i}, \\
g_{0}=-\sum_{i=1}^{3} b_{0 i}\left(h_{i}^{2}-h_{i-1}^{2}\right)\left(A_{2 i} d_{i} T^{2}-A_{1 i} d_{i} T\right), g_{1}=-\sum_{i=1}^{3} b_{0 i}\left(h_{i}^{2}-h_{i-1}^{2}\right)\left(A_{1 i}-2 A_{2 i} d_{i} T\right), \\
g_{2}=-\sum_{i=1}^{3} b_{0 i}^{2}\left(h_{i}^{2}-h_{i-1}^{2}\right)\left(2 A_{2 i} d_{i} T-A_{1 i}\right), g_{11}=-\sum_{i=1}^{3} b_{0 i}\left(h_{i}^{2}-h_{i-1}^{2}\right) A_{2 i}, \\
g_{22}=-\frac{1}{3} \sum_{i=1}^{3} b_{0 i}^{2}\left(h_{i}^{2}-h_{i-1}^{2}\right) A_{2 i}, g_{12}=\sum_{i=1}^{3} b_{0 i}^{2}\left(h_{i}^{2}-h_{i-1}^{2}\right) A_{2 i}, h_{0}=0 .
\end{gathered}
$$

The equations (15), (16) are second order polynomials with respect to $\frac{d u_{0}}{d x}$ and $\frac{d^{2} w_{0}}{d x^{2}}$.

From the conditions of fastening the rod, we obtain

$$
u(l)=w(0)=w(l)=0 .
$$

The further solution of the problem is reduced to the numerical solution of a system of two nonlinear algebraic equations with respect to two unknowns and the subsequent calculation of the deformation by formulas (6)-(7). Moreover, the deformation for each layer must be in the interval $-\varepsilon_{*_{i}}^{-} \leq \varepsilon \leq \varepsilon_{*_{i}}^{+}$.

This work is carried out with the partial financial support of RFBR grant (project №17-41210272).

\section{References}

1. A. V. Mishenko, Y. V. Nemirovskii, News of higher educational institutions. Construction, 4, 5-12 (2013)

2. P. Garsia, A.C. Esminella, M. N. C. Garsia, Composite. Part B: Engineering, 55, 528536 (2013)

3. Y. V. Nemirovskii, Proc. Conf. Fundamental and Applied Problems of Modern Mechanics, Tomsk, 288-290 (2006)

4. Y. V. Nemirovskii, A. I. Boltaev, Bulletin of BSTU named after V.G. Shukhov, 6, $125-$ 129 (2016)

5. Y. V. Nemirovskii, A. A. Baturin, News of higher educational institutions. Construction, 10, 82-93 (2015)

6. Y. A. Krus, News of higher educational institutions. Construction, 7, 113-122 (2008)

7. Y.V. Nemirovskii, A. V. Mishchenko, R. F. Terletskii, Journal of Mathematical Sciences, 223, 1, 87-102 (2017)

8. A. V. Gemlering, Calculation of rod systems (Stroitelstvo, Moskow, 1974)

9. P. A. Lukash Fundamentals of nonlinear construction mechanics (Stroitelstvo, Moskow, 1974) 
10. V. V. Petrov, Nonlinear incremental structural mechanics (Infra-Engineering, Moskow, 2014)

11. V. V. Adishev, A. G. Demeshkin, V. V. Rot, News of higher educational institutions. Construction, 3, 121-126 (2012)

12. V. O. Almazov, A. V. Zabegaev, N. N. Popov, S. V. Rastorguev, News of higher educational institutions. Construction, 11, 10-15 (1994) 